\title{
Treatment of lung adenocarcinoma brain metastases: what is the role of radiotherapy in the age of precision medicine?
}

\author{
Elizabeth J. Buss ${ }^{1}$, Tony J. C. Wang ${ }^{1,2}$ \\ ${ }^{1}$ Department of Radiation Oncology, ${ }^{2}$ Herbert Irving Comprehensive Cancer Center, Columbia University Irving Medical Center, New York, NY, USA \\ Correspondence to: Tony J. C. Wang. Department of Radiation Oncology, Columbia University Irving Medical Center, New York, NY 10032, USA. \\ Email: tjw2117@cumc.columbia.edu. \\ Provenance: This is an invited Editorial commissioned by Section Editor Jun Zhou, MD (Department of Nuclear Medicine, Zhongshan Hospital, \\ Fudan University, Shanghai, China). \\ Comment on: Press RH, Zhang C, Cassidy RJ, et al. Targeted sequencing and intracranial outcomes of patients with lung adenocarcinoma brain \\ metastases treated with radiotherapy. Cancer 2018;124:3586-95.
}

Submitted Nov 30, 2018. Accepted for publication Dec 14, 2018.

doi: $10.21037 /$ tlcr.2018.12.07

View this article at: http://dx.doi.org/10.21037/tlcr.2018.12.07

Lung cancer is the second most common type of cancer and the leading cause of cancer-related mortality (1). The discovery of oncogenic driver mutations and the development of immune checkpoint inhibitors has resulted in improved clinical outcomes for these patients. As systemic therapies continue to improve in the era of precision medicine, patients live longer and are thus at increased risk for brain metastases (2). Lung cancer, specifically non-small cell lung cancer (NSCLC), is the most common primary cancer associated with development of brain metastases with approximately $30-40 \%$ of affected patients developing brain metastases (1). Treatment of intracranial disease in this setting is multimodal and can include systemic therapy, surgery, stereotactic radiosurgery (SRS), whole brain radiotherapy (WBRT), and/or supportive care.

Molecular profiling of a lung cancer primary is standard practice and guides choice of first-line systemic therapy. The landscape of molecular alterations in a particular patient's tumor has strong implications for survival. The Lung-molGPA is a tool for estimating survival in patients with lung cancer and brain metastases and accounts for this association of gene alteration and survival. Presence of EGFR and $A L K$ gene alterations of the original primary lung tumor are considered positive prognostic factors for patients with NSCLC and brain metastases (2). Despite the connection between overall survival and the presence of a targetable mutation, analysis of molecular mutations is not a standard part of the decision-making paradigm for appropriate use of radiotherapy.

Press et al. recently conducted a study to examine the frequency and subtype of molecular alterations in patients with brain metastases from NSCLC and to identify markers associated with intracranial outcomes (3). In this retrospective study, 132 patients with metastatic NSCLC adenocarcinoma brain metastases and successful nextgeneration sequencing who were treated with intracranial radiotherapy were analyzed. The study demonstrated differences in outcomes for these patients based on molecular subtype of their primary tumor. Of particular clinical interest is their finding that patients with KRAS mutations and greater than six brain metastases have an increased risk of requiring salvage WBRT after having received upfront SRS for their intracranial disease. Such a finding has implications for the potential of molecular subtyping to guide radiotherapy decision making.

KRAS mutation is found in 25-30\% of lung adenocarcinomas and is the most common oncogenic genetic event in NSCLC. Despite incredible progress in biomarker-driven targeted therapy for other NSCLC oncogenic mutations and rearrangements such as EGFR, $A L K$ or ROS1, KRAS remains an elusive target for which there is currently no targeted therapy available. While many $K R A S$ targeted agents have been evaluated in clinical trials, none have shown survival benefit (4). Interestingly, KRAS positive NSCLC has been implicated as an independent predictor for the development of brain metastases in some 
studies (5).

For many years, the standard treatment for brain metastases was whole brain radiation therapy. As SRS became available in the 1980 s as a second option for treatment of brain metastases, questions emerged regarding optimal patient stratification and overall efficacy for each modality. Broadly speaking, many studies for these purposes divided patients into two subgroups based on number of brain metastases: limited and multiple, with limited defined as the presence of $1-3$ metastases and multiple defined as four or more. RTOG 9508 was a randomized trial that sought to investigate whether addition of SRS to WBRT could increase efficacy of treatment in patients with a limited number of brain metastases. The study found that addition of SRS to WBRT yields no difference in overall survival (6). Many later studies of patients with limited brain metastases again showed no overall survival benefit with WBRT and SRS versus SRS alone and additionally showed that SRS alone results in less neurocognitive deterioration at 3 months $(7,8)$. Because WBRT is associated with worse neurocognition and the same overall survival, SRS is often given upfront for intracranial disease with WBRT reserved as salvage therapy for treatment of subsequent disease progression. Many studies are currently ongoing investigating outcomes for patients with multiple brain metastases. Multiple series, however, show that volume of intracranial disease is likely more important than number of metastases in determining prognosis (9). And most recently, mounting evidence, including that from Press et al., has shown that molecular subtyping plays an important role in patient outcome as well $(3,10)$. In turn, one must ask if number of metastases is the best way to stratify patients, even initially, as appropriate candidates for SRS versus WBRT.

Currently, extensive and systematic analyses of the relationship between radiation efficacy and cancer genomics is lacking. This has prevented the movement of radiotherapy, a mainstay of anticancer treatment, into the age of precision medicine. Studies that identify frequent, potentially targetable, or prognostic genomic alterations that are correlated with the likelihood of response to radiation therapy are greatly needed. Press et al. is commended for taking a necessary step in this direction with their recent paper investigating the frequency and relationship of genetic aberrations in patients with NSCLC adenocarcinoma brain metastases and their response to treatment with radiotherapy. A greater understanding of the interaction between the cancer genome and radiation will allow for more individualized treatment of patients based on tumor characteristics in addition to anatomic origin and has the potential to enhance effectiveness of treatment and limit toxicity in the event that genetic complexity of tumor cells also regulates therapeutic response to radiation.

\section{Acknowledgements}

None.

\section{Footnote}

Conflicts of Interest: Dr. TJ Wang reports personal fees and non-financial support from AbbVie, non-financial support from Merck, personal fees from AstraZeneca, personal fees from Doximity, non-financial support from Novocure, personal fees and non-financial support from Elekta and personal fees from Wolters Kluwer, outside the submitted work. EJ Buss has no conflicts of interest to declare.

\section{References}

1. Nayak L, Lee EQ, Wen PY. Epidemiology of brain metastases. Curr Oncol Rep 2012;14:48-54.

2. Sperduto PW, Yang TJ, Beal K, et al. Estimating Survival in Patients With Lung Cancer and Brain Metastases: An Update of the Graded Prognostic Assessment for Lung Cancer Using Molecular Markers (Lung-molGPA). JAMA Oncol 2017;3:827-31.

3. Press RH, Zhang C, Cassidy RJ, et al. Targeted sequencing and intracranial outcomes of patients with lung adenocarcinoma brain metastases treated with radiotherapy. Cancer 2018;124:3586-95.

4. Kaufman J, Stinchcombe TE. Treatment of KRAS-Mutant Non-Small Cell Lung Cancer: The End of the Beginning for Targeted Therapies. JAMA 2017;317:1835-7.

5. Zhao N, Wilkerson MD, Shah U, et al. Alterations of LKB1 and KRAS and risk of brain metastasis: comprehensive characterization by mutation analysis, copy number, and gene expression in non-small-cell lung carcinoma. Lung Cancer 2014;86:255-61.

6. Andrews DW, Scott CB, Sperduto PW, et al. Whole brain radiation therapy with or without stereotactic radiosurgery boost for patients with one to three brain metastases: phase III results of the RTOG 9508 randomised trial. Lancet 2004;363:1665-72.

7. Brown PD, Jaeckle K, Ballman KV, et al. Effect of Radiosurgery Alone vs Radiosurgery With Whole Brain 
Radiation Therapy on Cognitive Function in Patients With 1 to 3 Brain Metastases: A Randomized Clinical Trial. JAMA 2016;316:401-9.

8. Chang EL, Wefel JS, Hess KR, et al. Neurocognition in patients with brain metastases treated with radiosurgery or radiosurgery plus whole-brain irradiation: a randomised controlled trial. Lancet Oncol 2009;10:1037-44.

9. Yamamoto M, Serizawa T, Shuto T, et al. Stereotactic

Cite this article as: Buss EJ, Wang TJ. Treatment of lung adenocarcinoma brain metastases: what is the role of radiotherapy in the age of precision medicine? Transl Lung Cancer Res 2018;7(Suppl 4):S318-S320. doi: 10.21037/ tlcr.2018.12.07 radiosurgery for patients with multiple brain metastases (JLGK0901): a multi-institutional prospective observational study. Lancet Oncol 2014;15:387-95.

10. Wang TJ, Saad S, Qureshi YH, et al. Does lung cancer mutation status and targeted therapy predict for outcomes and local control in the setting of brain metastases treated with radiation? Neuro Oncol 2015;17:1022-8. 